Pacific Journal of Mathematics

HOMOTOPIES AND INTERSECTION SEQUENCES 


\title{
HOMOTOPIES AND INTERSECTION SEQUENCES
}

\author{
J. R. QUINE
}

\begin{abstract}
For $\gamma_{t}: S^{1} \rightarrow \mathrm{C}$, a smooth homotopy of closed curves, the changing configuration of vertices and cusps is studied by considering the set in $I \times S^{1} \times S^{1}$ given by $\left(\gamma_{t}(z)-\gamma_{t}(\zeta)\right) /(z-\zeta)=0$. The main tool is oriented intersection theory from differential topology. The results relate to previous work by Whitney and Titus on normal curves and intersection sequences.
\end{abstract}

Consider a closed curve as a smooth map $\gamma: S^{1} \rightarrow \mathbf{C}$. Let $\gamma_{t}$ for $t \in I$ be a smooth homotopy of closed curves. A vertex of $\gamma_{t}$ is a point $w$ such that $w=\gamma_{t}(z)=\gamma_{t}(\zeta)$ for $z \neq \zeta$. A cusp is a point where the tangent vanishes and changes direction. Let $X=I \times S^{1} \times S^{1}$. We study the changing configuration of vertices and cusps of $\gamma_{t}$ by studying the set $Z=\{x \in X \mid G(x)=0\}$ where $G(t, z, \zeta)=\left(\gamma_{t}(z)-\gamma_{t}(\zeta)\right) /(z \cdot-\zeta)$, and the limiting value is taken when $z=\zeta$. If 0 is a regular value for $G$, then $Z$ has the structure of an oriented 1-submanifold of $X$. If for fixed $t, Z$ intersects $t \times S^{1} \times S^{1}$ transversely, then the oriented intersection gives a set of pairs in $S^{1} \times S^{1}$ with corresponding orientation numbers +1 or - 1. If $\gamma_{t}$ is a normal immersion, these pairs and their orientation numbers give the Titus intersection sequence of $\gamma_{t}$. The changes in the intersection sequence are reflected in the behavior of $Z$. If $Z$ crosses $I \times \Delta$, where $\Delta$ is the diagonal of $S^{1} \times S^{1}$, then we have a cusp and a change in the tangent winding number. The difference between the tangent winding numbers of $\gamma_{0}$ and $\gamma_{1}$ is just $N(Z, I \times \Delta)$, the total number of oriented intersections of $Z$ with $I \times \Delta$.

1. Intersection sequences. In the complex plane, let $S^{1}$ be the set $|z|=1$. Consider $S^{1}$ as a 1 -manifold with functions $\theta \rightarrow e^{i \theta}$ giving local coordinate systems. The tangent vector $d / d \theta$ is defined independently of the choice of coordinate system. On $T\left(S^{1}\right)$, the tangent space, let $d / d \theta$ give the positive orientation at each point. This gives $S^{1}$ the structure of an oriented 1-manifold.

Suppose $\gamma: S^{1} \rightarrow \mathbf{C}$ is a smooth $\left(C^{\infty}\right)$ map. Let $\beta(z)=(d \gamma / d \theta)(z)$ be the tangent at $\gamma(z)$. Let $S^{1} \times S^{1}=Y$ and let the maps $(\theta, \phi) \rightarrow\left(e^{i \theta}, e^{i \phi}\right)$ give local coordinate systems for $Y$. Let $S^{1} \times S^{1}$ have the product orientation, i.e., $T\left(S^{1} \times S^{1}\right)$ has positive orientation given by the ordered basis $\{\partial / \partial \theta, \partial / \partial \phi\}$ at each point. Let $\Delta \subseteq Y=\{(z, \zeta) \mid z=\zeta\}$.

Let $\theta \rightarrow\left(e^{i \theta}, e^{i \theta}\right)$ be local coordinate systems on $\Delta$ and let positive 
orientation be given on $\Delta$ by $d / d \theta$. Thus $\Delta$ is an oriented 1-submanifold of $Y$. Now we define $g: Y \rightarrow \mathrm{C}$ as follows

$$
g(z, \zeta)= \begin{cases}\frac{\gamma(z)-\gamma(\zeta)}{z-\zeta}, & z \neq \zeta \\ \frac{-i \beta(z)}{z}, & z=\zeta\end{cases}
$$

We can check that $g$ is a smooth function on $Y$.

Letting $y=(z, \zeta)$, we compute that for $y \in g^{-1}(0)$ we have

$$
d g_{y}= \begin{cases}\frac{\beta(z) d \theta-\beta(\zeta) d \phi}{z-\zeta}, & z \neq \zeta \\ \frac{1}{i z} \frac{d \beta}{d \theta}(z)(d \theta+d \phi), & z=\zeta .\end{cases}
$$

Now let $y=(z, \zeta) \in g^{-1}(0)$, and consider $d g_{y}$ as a linear map from $T_{y}(Y)$ to $T_{0}(\mathbf{C})$. Then from $(1)$ :

(a) If $z \neq \zeta$, then $d g_{y}$ has rank 2 iff the tangents $\beta(z)$ and $\beta(\zeta)$ are linearly independent. In this case, $d g_{y}$ preserves orientation iff $\{\beta(z),-\beta(\zeta)\}$ is a positively oriented basis of $\mathbf{C}$ (where $\mathbf{C}$ has the usual orientation).

(b) If $z=\zeta$, then $\beta(z)=0$ and $d g_{y}$ has rank 1 iff $(d \beta / d \theta)(z) \neq 0$. Otherwise $d g_{y}$ has rank 0 . We may check that if $(d \beta / d \theta)(z) \neq 0$, then there is a cusp at $\gamma(z)$ and the limiting tangential directions at $\gamma(z)$ are the directions of $\pm(d \beta / d \theta)(z)$.

The point $0 \in \mathbf{C}$ is said to be a regular value for $g$ if $d g_{y}$ has rank 2 at every point of $g^{-1}(0)$. By remarks (a) and (b) above we see that 0 is a regular value for $g$ iff $\gamma$ is an immersion $\left(\beta(z) \neq 0\right.$ for $\left.z \in S^{1}\right)$, and the tangents $\beta(z)$ and $\beta(\zeta)$ are linearly independent for each point $(z, \zeta) \in$ $g^{-1}(0)$. Also if 0 is a regular value of $g, g^{-1}(0)$ is a finite subset of the compact set $Y$ (a torus). In this case if $y \in g^{-1}(0)$ we set $\lambda(y)=+1$ if $d g_{y}$ preserves orientation and $\lambda(y)=-1$ if $d g_{y}$ reverses orientation. We say that $g^{-1}(0)$ with the sign $\lambda$ gives the set of signed intersection pairs for $\gamma$.

We say that $\gamma$ is a normal immersion if $\gamma$ is an immersion, each point of $\mathbf{C}$ has at most two preimages under $\gamma$, and the tangents are linearly independent at each double point. Another way to say this is that 0 is a regular value for $g$, and projection on the first coordinate is one-to-one on $g^{-1}(0)$. $\left(g^{-1}(0)\right.$ as a set of ordered pairs is a function.) If $\gamma$ is a normal immersion, let $\left\{z_{1}, \cdots, z_{2 n}\right\}$ be the preimages under $\gamma$ of the double points, numbered sequentially along $S^{1}$ in a counterclockwise direction from a point $z_{0}$ on $S^{1}$, not a preimage of a double point. Then $g^{-1}(0)$ defines an involution * on the integers $1, \cdots, 2 n$, such that $\left(z_{l}, z_{j^{*}}\right) \in g^{-1}(0)$ 
for $j=1, \cdots, 2 n$. Now define the sign $\nu$ by $\nu(j)=-\lambda\left(\left(z_{,}, z_{j^{*}}\right)\right)$. We say that the involution ${ }^{*}$ together with the sign $\nu$ defines the intersection sequence of $\gamma$ with respect to $z_{0}$. Usually $z_{0}$ is chosen so that $\gamma\left(z_{0}\right)$ is on the outer boundary, i.e., the boundary of the component of $\mathbf{C}-\gamma\left(S^{1}\right)$ containing $\infty$. In this case $\nu$ and ${ }^{*}$ give the Titus intersection sequence (see Titus [5] or Francis [1]). We remark that signed intersection pairs are defined if 0 is a regular value for $g$. To define the intersection sequence also, we need in addition that $g^{-1}(0)$ is a function.

2. The fundamental theorem. In this context, we would like to prove what we call the fundamental theorem on intersection sequences. The use of intersection pairs allows a slightly more general statement than that of Whitney [6] and Titus [5]. Let $\gamma$ be a normal immersion and let $[\gamma]$ denote the image of $\gamma$. For $a \in \mathbf{C}-\gamma(R)$ we define $j_{a}$ on $S_{a}=S^{1}-\gamma^{-1}(a)$ by $j_{a}=(\gamma-a) /|\gamma-a|$. We define

$$
\omega(\gamma, a)=\frac{1}{2 \pi i} \int_{S_{a}} \frac{d j_{a}}{j_{a}}
$$

If $a \notin \gamma$, this is just the winding number of $\gamma$ about $a$. If $a \in[\gamma]$, we may check that $\omega(\gamma, a)$ is the average of the winding numbers of $\gamma$ on the components near $\gamma(a)$.

Now, for fixed $z_{0} \in S^{1}$, consider $z_{0} \times S^{1}$ and $S^{1} \times z_{0}$ as subsets of $Y$. Let $\theta \rightarrow\left(z_{0}, e^{i \theta}\right)$ and $\phi \rightarrow\left(e^{i \phi}, z_{0}\right)$ be coordinate systems on $z_{0} \times S^{1}$ and let these define the orientations. Thus, $z_{0} \times S^{1}$ and $S^{1} \times z_{0}$ have the structures of oriented 1-submanifolds of $Y$. Now $W=z_{0} \times S^{1}$ $+S^{1} \times z_{0}-\Delta$ divides the torus $Y$ into 2 simply connected 2-manifolds with boundary, $Y^{+}$and $Y^{-}$. Here $Y^{+}$denotes the one for which $W$ is a positively oriented boundary and $Y^{-}$the one for which $W$ is a negatively oriented boundary (see Fig. 1).

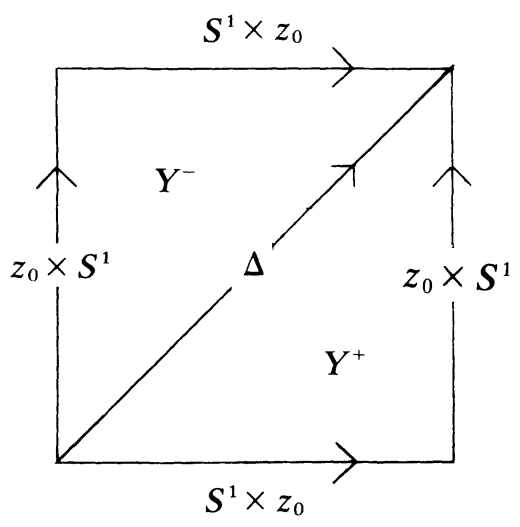

FIG. 1 
If $\gamma$ is an immersion, and $\beta=d \gamma / d \theta$ is the tangent, then the tangent winding number, twn $\gamma$, is defined to be

$$
\frac{1}{2 \pi i} \int_{S^{1}} \frac{d \beta}{\beta}
$$

We now have

THEOREM 1 (Titus-Whitney). If 0 is a regular value for $g, z_{0} \in S^{1}$, and $Y^{+}$is the oriented 2-submanifold of $S^{1} \times S^{1}$ with positively oriented boundary $z_{0} \times S^{1}+S^{1} \times z_{0}-\Delta$, then

$$
\text { twn } \gamma=-\sum_{y \in Y^{+} \cap g^{-1}(0)} \lambda(y)+2 \omega\left(\gamma, \gamma\left(z_{0}\right)\right) \text {. }
$$

Proof. Let $g^{-1}(0) \cap Y^{+}=\left\{y_{1}, \cdots, y_{n}\right\}$. Let $D_{1}, \cdots, D_{n}$ be closed disjoint coordinate discs in $Y^{+}$such that $D_{j} \cap g^{-1}(0)=Y_{j}$ for $j=$ $1, \cdots, n$. Let these have orientation inherited from $Y$ and let $\partial D$, be the oriented boundary of $D_{j}$ for $j=1, \cdots, n$. Recall that for $j=1, \cdots, n$, $\lambda\left(y_{j}\right)=+1$ iff $d g$ preserves orientation at $y_{j}$. Therefore we may choose each $D$, so that

$$
\frac{1}{2 \pi i} \int_{D,} \frac{d g}{g}=\lambda\left(y_{J}\right)
$$

Now $d g / g$ is closed on $Y^{+}-\bigcup_{j=1}^{n} D_{j}$ so the integral of $d g / g$ over its boundary is 0 . The boundary is the cycle $z_{0} \times S^{1}+S^{1} \times z_{0}-\Delta-\sum_{j=1}^{n} \partial D_{j}$. From the definition of $g$,

$$
\frac{1}{2 \pi i} \int_{z_{0} \times S^{1}} \frac{d g}{g}=\frac{1}{2 \pi i} \int_{S^{1} \times z_{0}} \frac{d g}{g}=\omega\left(\gamma, \gamma\left(z_{0}\right)\right)-1 / 2
$$

and $(1 / 2 \pi i) \int_{\Delta} d g / g=t w n \gamma-1$. The theorem now follows. We remark that if $\gamma\left(z_{0}\right)$ is on the outer boundary of $\gamma$ and its image is not a multiple point of $\gamma$, then $\omega\left(\gamma, \gamma\left(z_{0}\right)\right)= \pm \frac{1}{2}$. In this case, if $\gamma$ is a normal immersion, then Theorem 1 is Lemma 3 of Titus [5].

3. Homotopies. Let $I=[0,1]$ considered as an oriented 1manifold with boundary having the usual orientation. Let $I \times S^{1}$ be an oriented 2-manifold with boundary with the product orientation. A smooth map $F: I \times S^{1} \rightarrow \mathbf{C}$ is called a homotopy. Let $\gamma_{t}(z)=F(t, z)$ and $\beta_{t}(z)=\left(d \gamma_{t} / d \theta\right)(z)$. Let $X=I \times S^{1} \times S^{1}$ and $Y_{t}=t \times S^{1} \times S^{1} \subseteq X$ where both are given the product orientations. Define $G: X \rightarrow \mathbf{C}$ by 


$$
G(t, z, \zeta)= \begin{cases}\frac{F(t, z)-F(t, \zeta)}{z-\zeta}, & z \neq \zeta \\ \frac{-i \beta_{t}(z)}{z}, & z=\zeta\end{cases}
$$

Define $g_{t}: S^{1} \times S^{1} \rightarrow$ C by $g_{t}(z, \zeta)=G(t, z, \zeta)$. Let $Z=\{x \in X \mid G(x)=$ 0 . We say 0 is a regular value for $G$ if $d G$ has rank 2 everywhere on $Z$. In this case, by the implicit function theorem, $Z$ has the structure of a 1 -submanifold of $X$, with boundary. We intend to study the change in the intersection sequence under the homotopy $F$ by looking at the smooth manifold $Z \subseteq X$, therefore we will make the assumption that 0 is a regular value for $G$.

To justify this assumption, we prove the following lemma.

LEMMA 1. If $F(t, z)=\gamma_{t}(z)$ is a smooth homotopy of closed curves and $\quad G(F): I \times S^{1} \times S^{1} \rightarrow \mathbf{C}$ is defined by $G(F)(t, z, \zeta)=$ $(F(t, z)-F(t, \zeta)) /(z-\zeta)$, then $F$ may be deformed by an arbitrarily small amount into a homotopy $F$ for which 0 is a regular value for $G(F)$.

Proof. Let $D$ be the open disc $|w|<1$. For $w \in D$, define $F_{w}(t, z)=$ $F(t, z)+w z$. Note that $F_{0}(t, z)=F(t, z)$. Then $G\left(F_{w}\right)(t, z, \zeta)=$ $G(F)(t, z, \zeta)+w$. Clearly the map $(t, z, \zeta, w) \rightarrow G\left(F_{w}\right)(t, z, \zeta)+w$ from $\left(I \times S^{1} \times S^{1}\right) \times D$ to $\mathrm{C}$ is a submersion, and therefore 0 is a regular value for this function. By the transversality theorem (Guillemin and Pollack [3] p. 68), 0 is a regular value of $G\left(F_{w}\right)$ for almost all $w \in D$. This proves the lemma.

4. The orientation on $Z$. Assume that 0 is a regular value of $G$ so that $Z$ is a 1 -manifold with boundary. We will define an orientation on $Z$ such that we get a set of signed intersection pairs for $\gamma_{t}$ by intersecting $Z$ with $Y_{t}$. At each intersection point, the sign will be defined by the orientation of $Z$ and $Y_{t}$.

First we indicate how to define a direct sum orientation on vector spaces. If $V$ and $W$ are oriented subspaces of a vector space and if the ordered bases $\left\{v_{1}, \cdots, v_{n}\right\}$ and $\left\{w_{1}, \cdots, w_{m}\right\}$ define positive orientation of $V$ and $W$ respectively, then the sum orientation on $V \oplus W$ (in that order) is defined by the ordered basis $\left\{v_{1}, \cdots, v_{n}, w_{1}, \cdots, w_{m}\right\}$.

We now orient $Z$ as follows: If $x \in Z$, write $T_{x}(X)=T_{x}(Z) \oplus H$. Then $d G_{x}: H \rightarrow T_{0}(\mathbf{C})$ and the mapping is a vector space isomorphism. In a natural way, this isomorphism induces an orientation on $H$ from the usual orientation on $T_{0}(\mathbf{C})$. We now choose an orientation on $T_{x}(Z)$ so that the sum orientation agrees with the prescribed orientation on $T_{x}(X)$. In this way $Z$ is given the structure of an oriented 1-manifold. 
Now as before let $Y_{t}=t \times S^{1} \times S^{1}$ with the product orientation. Suppose $x=(t, z, \zeta) \in Z \cap Y_{t}$ and $d\left(g_{t}\right)_{(z, \zeta)}$ preserves orientation. Then $d G_{x}$ preserves orientation on $T_{x}\left(Y_{t}\right)$. Now we can can write $T_{x}(X)=$ $T_{x}(Z) \oplus T_{x}\left(Y_{t}\right)$ where by definition, the orientations sum to the prescribed orientation on $T_{x}(X)$. In this case the intersection number at $x \in Z \cap Y_{t}$ is said to be +1 (here the order in which we list $Z$ and $Y_{t}$ is important (see Guillemin and Pollack [3])). Likewise if $d\left(g_{t}\right)_{(z, \zeta)}$ reverses orientation, the intersection number of $x \in Z \cap Y_{t}$ is -1 . Thus if $d\left(g_{t}\right)_{(z, \zeta)}$ has rank 2 at each point $x \in Z$ then the set $Z \cap Y_{t}$ along with the intersection number at each point gives us the set of signed intersection pairs for $\gamma_{t}$.

5. The change in the intersection sequences. The configuration of the oriented 1-manifold $Z$ as a submanifold of $X$ indicates how the intersection pairs and the intersection sequence changes under the homotopy $F$. (We may take the intersection sequence with respect to a continuously moving point whose image stays on the outer boundary.) We mention here only some general considerations:

(a) $Z$ is symmetric with respect to $I \times \Delta$, i.e., $(t, z, \zeta) \in Z$ iff $(t, \zeta, z) \in Z$.

(b) The components of $Z$ are oriented 1-manifolds homeomorphic to either $S^{1}$ or $I$ (see Guillemin and Pollack [3] Appendix 2 or Milnor [4] Appendix).

(c) Each component either crosses $I \times \Delta$ and is symmetric with respect to $I \times \Delta$ or has another component symmetric to it with respect to $I \times \Delta$ (see Fig. 2).

(d) When a component of $Z$ crosses $I \times \Delta$ we have a change in twn $\gamma_{t}$. We will describe this fully in the next section.

(e) Each component of $Z$ represents a continuously moving vertex on $\gamma_{t}$. Components homeomorphic to $I$ and joining points on $Y_{0}$ represent vertices lost in homotopy. Components homeomorphic to $I$ and joining points in $Y_{1}$ represent vertices gained.

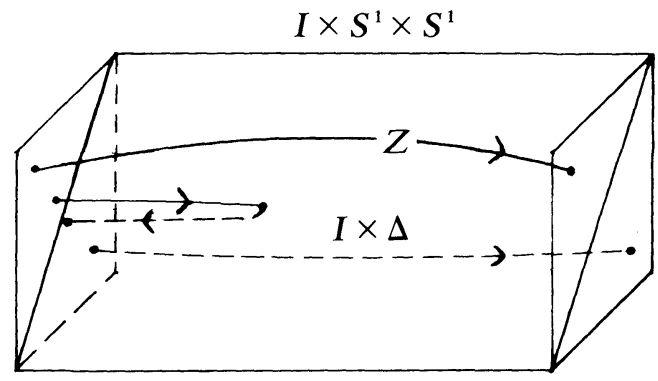


Finally, suppose that $\Pi: X=I \times S^{1} \times S^{1} \rightarrow I \times S^{1}$ is the projection on the first two coordinates. Then $\Pi(Z) \subseteq I \times S^{1}$ consists of smooth curves. If the intersection sequence of $\gamma_{t}$ changes at $t_{0}$, then either some vertices coincide, in which case $\Pi(Z)$ crosses itself at a point $\left(t_{0}, z\right)$ or else a vertex appears or disappears, in which case the real valued function $t$ on $Z$ has a relative maximum or minimum at a point $\left(t_{0}, z, \zeta\right)$ on $Z$.

6. Change in twn $\gamma_{t^{\bullet}}$ Let $I \times \Delta \subseteq X$ have the usual product orientation. Say $Z$ intersects $I \times \Delta$ transversely if $T_{x}(Z) \oplus T_{x}(I \times \Delta)=$ $T_{x}(X)$ at each point $x \in Z \cap(I \times \Delta)$. Let $N(Z, I \times \Delta)$ be the intersection multiplicity of $Z$ with $I \times \Delta$, i.e., the sum of the intersection numbers at points of $Z \cap(I \times \Delta)$. We prove the following theorem concerning the change in twn $\gamma_{t}$ for the homotopy.

THEOREM 2. If $Z$ intersects $I \times \Delta$ transversely, then twn $\gamma_{1}-$ twn $\gamma_{0}=N(Z, I \times \Delta)$.

Proof. Let $Z \cap(I \times \Delta)=\left\{y_{1}, \cdots, y_{n}\right\}$. At $y=y_{j}$ write $T_{y}(X)=$ $T_{y}(Z) \oplus T_{y}(I \times \Delta)$. By definition of the intersection number at $y_{j}$ and by definition of the orientation of $Z$ we see that the intersection number at $y=y_{1}$ is +1 iff $d G_{y}$ preserves orientation on $T_{y}(I \times \Delta)$. Now we can choose closed disjoint coordinate discs $D_{1}, \cdots, D_{n}$ in $I \times \Delta$ such that $D_{l} \cap Z=y_{l}$ for $j=1, \cdots, n$ and $(1 / 2 \pi i) \int_{\partial D_{l}} d G / G=$ the orientation number at $y_{j} \in Z \cap(I \times \Delta)$. Now $d G / G$ is closed on $I \times \Delta-\bigcap_{j=1}^{n} D$, and the boundary is $1 \times \Delta-0 \times \Delta-\sum_{j=1}^{n} \partial D_{j}$. Now $(1 / 2 \pi i) \int_{0 \times \Delta} d G / G=t w n \gamma_{0}$ and $(1 / 2 \pi i) \int_{1 \times \Delta} d G / G=t w n \gamma_{1}$, and integration of $d G / G$ over the boundary gives 0 . This proves the theorem.

We have the following well-known:

COROllaRy 1. Regular homotopies preserve the tangent winding number.

Proof. In this case $Z \cap(I \times \Delta)=\varnothing$.

Finally, we remark that the fundamental theorem of Titus and Whitney becomes in this context:

THEOREM 3. Suppose for fixed $t \in I$ and $z_{0} \in S^{1}, Y_{t}^{+}$is the oriented submanifold of $I \times S^{1} \times S^{1}$ with positively oriented boundary $t \times z_{0} \times S^{1}+$ $t \times S^{1} \times z_{0}-t \times \Delta$. If $Z$ intersects $Y_{t}^{+}$transversely, 


$$
N\left(Z, Y_{t}^{+}\right)=t w n \gamma_{t}-2 \omega\left(\gamma_{t}, \gamma_{t}\left(z_{0}\right)\right)
$$

Proof. We observe that if $\dot{x}=(t, z, \zeta) \in Z \cap Y_{t}$ then the intersection number is +1 iff $d\left(g_{t}\right)_{(z, \zeta)}$ preserves orientation. Now the theorem follows from Theorem 1 .

\section{REFERENCES}

1. G. Francis, Null genus realizability criterion for abstract intersection sequences, J. Combinatorial Theory, 7 (1969), 331-341.

2. _- Titus' homotopies of normal curves, Proc. Amer. Math. Soc., 30 (1971), 511-518.

3. V. Guillemin and A. Pollack, Differential Topology, Prentice-Hall, Inc., Englewood Cliffs, New Jersey, 1974.

4. J. Milnor, Topology from the Differentiable Viewpoint, The University Press of Virginia, Charlottesville, 1965.

5. C. J. Titus, A theory of normal curves and some applications, Pacific J. Math., 10 (1960), 1083-1096.

6. H. Whitney, On regular closed curves in the plane, Comp. Math., 4 (1937), 276-284.

Received November 5, 1975 and in revised form February 24, 1976.

THE Florida STATE University 



\section{Pacific Journal of Mathematics}

Vol. 64, No. 1

May, 1976

Walter Allegretto, Nonoscillation theory of elliptic equations of order $2 n \ldots \ldots \quad 1$

Bruce Allem Anderson, Sequencings and starters.................. 17

Friedrich-Wilhelm Bauer, A shape theory with singular homology .......... 25

John Kelly Beem, Characterizing Finsler spaces which are

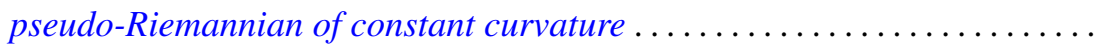

Dennis K. Burke and Ernest A. Michael, On certain point-countable

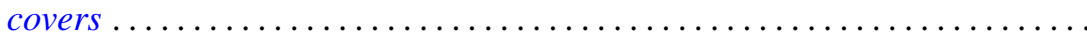

Robert Chen, A generalization of a theorem of Chacon ............... 93

Francis H. Clarke, On the inverse function theorem ................ 97

James Bryan Collier, The dual of a space with the Radon-Nikodým

property ....................................... 103

John E. Cruthirds, Infinite Galois theory for commutative rings ............ 107

Artatrana Dash, Joint essential spectra......................... 119

Robert M. DeVos, Subsequences and rearrangements of sequences in FK

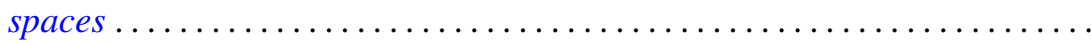

Geoffrey Fox and Pedro Morales, Non-Hausdorff multifunction generalization

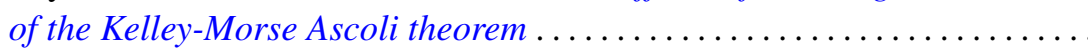

Richard Joseph Fleming, Jerome A. Goldstein and James E. Jamison, One

parameter groups of isometries on certain Banach spaces.............

Robert David Gulliver, II, Finiteness of the ramified set for branched

immersions of surfaces

Kenneth Hardy and István Juhász, Normality and the weak cb property ...... 167

C. A. Hayes, Derivation of the integrals of $L^{(q)}$-functions.

Frederic Timothy Howard, Roots of the Euler polynomials .

Robert Edward Jamison, II, Richard O'Brien and Peter Drummond Taylor, On

embedding a compact convex set into a locally convex topological vector space ....................................

Andrew Lelek, An example of a simple triod with surjective span smaller than span ...

Janet E. Mills, Certain congruences on orthodox semigroups

Donald J. Newman and A. R. Reddy, Rational approximation of $e^{-x}$ on the positive real axis.

John Robert Quine, Jr., Homotopies and intersection sequences ...

Nambury Sitarama Raju, Periodic Jacobi-Perron algorithms and fundamental units ....

Herbert Silverman, Convexity theorems for subclasses of univalent functions. . .

Charles Frederick Wells, Centralizers of transitive semigroup actions and endomorphisms of trees.........................

Volker Wrobel, Spectral approximation theorems in locally convex spaces ..................... 